\title{
Numerical simulation of collision between two gelled crude oil particles in a pipe
}

\author{
LI Xiaoqing ${ }^{1, a}$, LIU Xiaoyan ${ }^{2, b}$, LI Youhang ${ }^{3, c}$ \\ ${ }^{1}$ School of Civil Engineering, Northeast Petroleum University, Daqing ,163318, China \\ 2 Heilongjiang Key Laboratory of Disaster Prevention, Mitigation and Protection Engineering, \\ Daqing ,163318, China \\ ${ }^{3}$ Offshore Oil Engineering Corp, Tianjin,300452,China \\ aLi964499@126.com, bLiu_xydq@163.com, c1028782409@qq.com
}

\begin{abstract}
Keywords: oil/water two phase flow; gelled oil; collision, numerical simulation Abstract.In the hydraulic suspension conveying process, different size particles released from different positions into the flow field due to the different trajectories of collision, and the collision of gelled crude oil particles will lead to a large number of aggregated particles, is not conducive to the normal operation of hydraulic suspension conveying process. In this paper, the collision of gelled crude oil particles in the process of hydraulic suspension transportation is studied based on the principle of moving trajectories of gelled crude oil particles. According to the intersection principle of two particle trajectories and ignoring the acceleration process of particles, a collision model of two particles is established. The model is calculated by Matlab, and the calculated results are compared with the Fluent simulation results.
\end{abstract}

\section{Introduction}

Hydraulic suspension conveying is gathering a new kind of oil gas water multiphase flow, can be safe in crude oil gathering point, energy saving effect is obvious. In the hydraulic suspension conveying process of gelled crude oil by fluidization device is processed into plastic particles, mixing temperature at or below freezing point near the water, depending on the water carrying gelled crude oil particles for hydraulic suspension conveying. It is of great significance to master the movement law of gelled crude oil particles in hydraulic suspension to promote the development of this technology. In the process of hydraulic suspension transport, the particle size and release position have a significant influence on the trajectory of the particles. Particles with different particle sizes can enter into the flow field from different release locations, and may collide due to different trajectories. The collision of gelled crude oil particles will lead to a large number of particles gathering, which is not conducive to the normal operation of the hydraulic suspension transportation process.

Some basic problems in the study of multiphase flow can be divided into three large pieces of [1], particle particle interaction, particle fluid interaction and particle wall interaction. This paper is mainly aimed at the study of particle particle interaction. In view of the collision rule of particles in two phase flow, scholars used different methods to study, including Eulerian Euler method, namely the two fluid model, while the Euler Lagrange method, namely the discrete particle model [2-6]. In addition, the description of the dispersed phase is divided into random method and deterministic method. The stochastic model, such as the direct simulation Monte Carlo method, the deterministic model such as the soft ball model and the hard ball model of the discrete element method, such as [7-10]. In this paper, we simulate two trajectories of gelled crude oil particles and observe whether there are track crossover to study the collision between two particles. During the research process, particles are simplified as particles.

\section{Establishment of Mathematical Model for Collision between Two Gelled Crude Oil Particles}

The movement of dispersed phase gelled oil particles in the flow field is the synthesis of two movements: one is the translational motion with the fluid (continuous phase); the other is the floating motion itself under the action of the driving force of density difference. The movement process of 
gelled crude oil particles in the radial direction of the acceleration process and including uniform process, particles in flow field, the buoyancy is greater than gravity, accelerated motion in the vertical direction, but the time is short, then the particles do uniform motion in the vertical direction, so from the macro motion process, motion time and gelled crude oil particles float height is linear. If we ignore the acceleration process of particles, the floating velocity of particles at different locations depends only on the density difference, viscosity and particle size of dispersed phase and continuous phase.

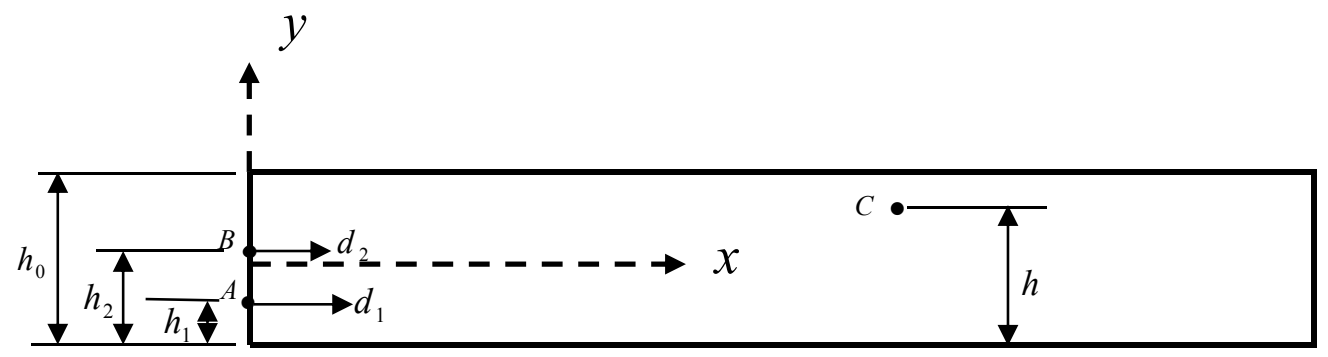

Figure 1 Schematic diagram of collision between two gelled crude oil particles

When the particle B is delayed to the particle A into the flow field, the two particles collide at C, as shown in Figure 1. The basic relationship between the parameters should be established by using the geometric trajectory of the crude oil particles to consider the collisions of particles.

Floatation speed of the gelled crude oil particles in the flow field is as follows:

$$
u_{s}=\frac{\rho_{w}-\rho_{o}}{18 \mu} g d^{2}
$$

For different positions (A and B), different time 0 and released gelled crude oil particles, in the C point collision, the vertical height should meet the relationship:

$$
t_{0}=\frac{18 \mu}{\left(\rho_{w}-\rho_{o}\right) g}\left(\frac{h-h_{1}}{d_{1}^{2}}-\frac{h-h_{2}}{d_{2}^{2}}\right) \quad\left(d_{2}<d_{1}, h_{1}<h_{2}\right)
$$

Velocity distribution law of the turbulent flow field is as follows:

$$
u_{x}=u_{\max }\left(1-\frac{|y|}{h_{0} / 2}\right)^{\frac{1}{6.6}}=u_{\max }\left(\frac{h_{0}-2|y|}{h_{0}}\right)^{0.15}
$$

Reference to the horizontal displacement and time of two gelled crude particles collisions, the following conditions should be satisfied.

$$
\int_{0}^{t}\left[u_{\max }\left(\frac{h_{0}-2\left|h_{1}+\frac{\left(\rho_{w}-\rho_{o}\right)}{18 \mu} g d_{1}^{2} t\right|}{h_{0}}\right)^{0.15} d t\right]=\int_{t_{0}}^{t}\left[u_{\max }\left(\frac{h_{0}-2\left|h_{2}+\frac{\left(\rho_{w}-\rho_{o}\right)}{18 \mu} g d_{2}^{2}\left(t-t_{0}\right)\right|}{h_{0}}\right)^{0.15} d t\right]
$$

Therefore, two gelled crude particles are moving in the flow field, and if the solution of (2) and (3) can be met at the same time, the two particles collide. 


\section{Comparison of Calculation Results and Simulation Results}

The mathematical model of gelled crude oil particle collision is established in this paper, is set up in the particle acceleration process under the condition of neglect, has certain limitations, because of the complexity of the collision, so far almost no test data can be used to express the relationship between the two, so the collision of gelled crude oil, this paper calculated by Matlab and the Fluent simulation analysis. The basic parameters are as follows: $\mu=10^{-3} \mathrm{~Pa} \cdot \mathrm{s}, \quad \rho_{w}=1000 \mathrm{~kg} / \mathrm{m}^{3}, \quad \rho_{o}=840 \mathrm{~kg} / \mathrm{m}^{3}$, $h_{0}=0.053 \mathrm{~m}, \quad u_{\max }=0.6 \mathrm{~m} / \mathrm{s}$. The small particle size gelled crude oil particles are released at the same time at the top of the large particle size gelled crude oil particles. $d_{1}=4 \mathrm{~mm}, h_{1}=0.0135 \mathrm{~m}, d_{2}=2 \mathrm{~mm}$, $h_{2}=0.0165 \mathrm{~m}$. The DPM model combined with UDF is used to simulate the motion law of particles in gelled crude oil, and the trajectories of two small particles in the flow field are obtained, as shown in Figure 2. It can be seen that the particle size $4 \mathrm{~mm}$ of large particles flow into the flow field from the $0.013 \mathrm{~m}$ below the pipe center, and the small particle size $2 \mathrm{~mm}$ is flowing into the flow field from the $0.01 \mathrm{M}$ below the pipe center. The trajectories of two particles appear to cross at $(0.097 \mathrm{~m},-0.0045 \mathrm{~m})$. If particles collide, they must arrive at the location of intersection at the same time.

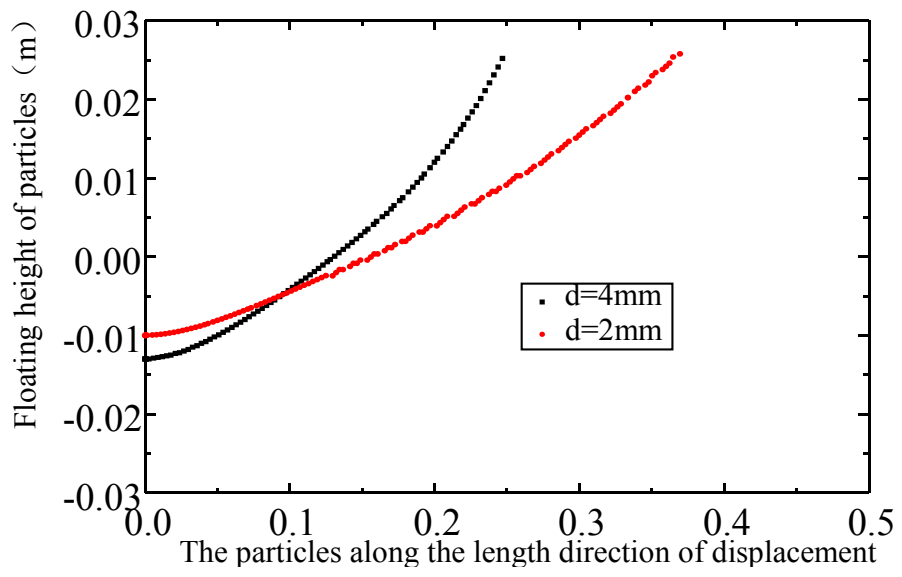

Fig. 2 The trajectories of two gelled crude oil particles

In order to study whether the time of the location of two particles arriving at the intersection point is the same, the relationship between particle horizontal displacement and floating height and time of movement is also plotted respectively, and the results are shown in figures 3 and 4 respectively.

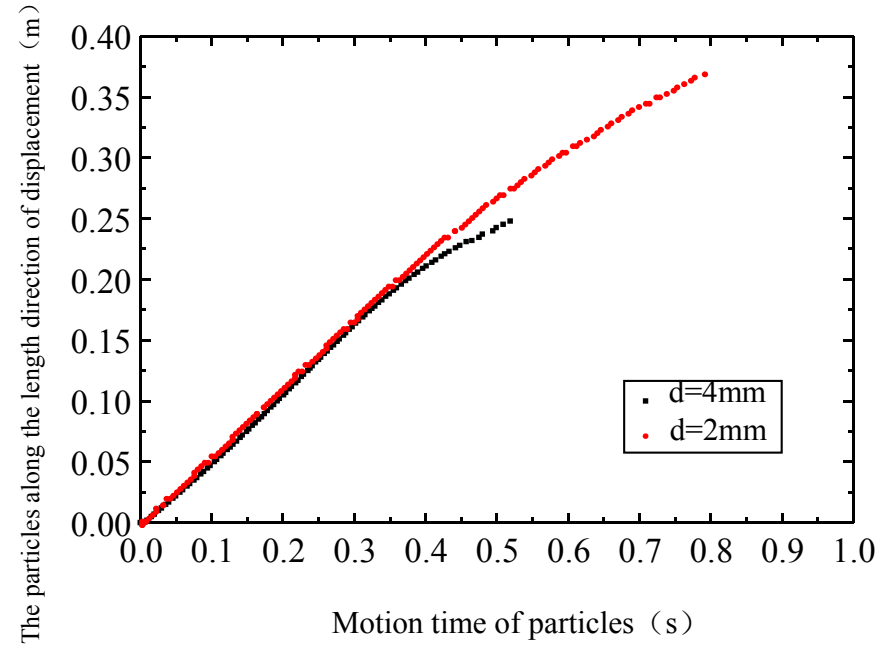

Fig.3 Variation of particle displacement along the length direction with time 
As shown in Figure 3, the particle size of $4 \mathrm{~mm}$ and the size of the change of $2 \mathrm{~mm}$ two gelled crude oil particle length in $0-0.3 \mathrm{~s}$ direction displacement is almost identical with the movement time. When the mobile particles along the length direction of $0.097 \mathrm{~m}$ movement needed for $0.20 \mathrm{~s}$, small particles move along the length direction of $0.097 \mathrm{~m}$ time required for $0.20 \mathrm{~s}$, they are the same, so the two particles in the horizontal direction at the same time to cross point.

After the $4 \mathrm{~mm}$ gelled crude particle entered the flow field under the $2 \mathrm{~mm}$ particle, the large particles gradually chased small particles at the height of the floating rise. When the large particles move to $0.0045 \mathrm{~m}$ below the center of the pipeline, the motion time is $0.20 \mathrm{~s}$, and the movement time required for the small particles moving to the same position is $0.20 \mathrm{~s}$. The two are the same, so the two particles reach the intersection point at the same time in the vertical direction. Through the above analysis can be drawn, the particle size of $4 \mathrm{~mm}$, distance from the center of pipeline $0.013 \mathrm{~m}$ into the flow field, small particle size $2 \mathrm{~mm}$, distance from the center of pipeline $0.01 \mathrm{M}$ into the field, two of gelled crude oil particles at the trajectory intersection in the $0.20 \mathrm{~s}(0.097 \mathrm{~m},-0.0045 \mathrm{~m})$, the collision of two particles. Matlab is used to solve the equation (2) and (3), and the basic parameters are substituted into the equation. After solving, two particles collision time is $=0.20 \mathrm{~s}$, and the location of collision is $(0.099 \mathrm{~m},-0.0054 \mathrm{~m})$. The collision position of two particles and calculated the $(0.097 \mathrm{~m}$, $-0.0045 \mathrm{~m}$ ) compared to the horizontal position is almost the same, there is a rise in the floating point error of height, analysis that the collision model is neglected in acceleration in the $\mathrm{Y}$ direction of particles on the set up, and the simulation calculation of particle acceleration process, $y$ so the displacement in the initial stage of particle by the model calculation than the simulation results.

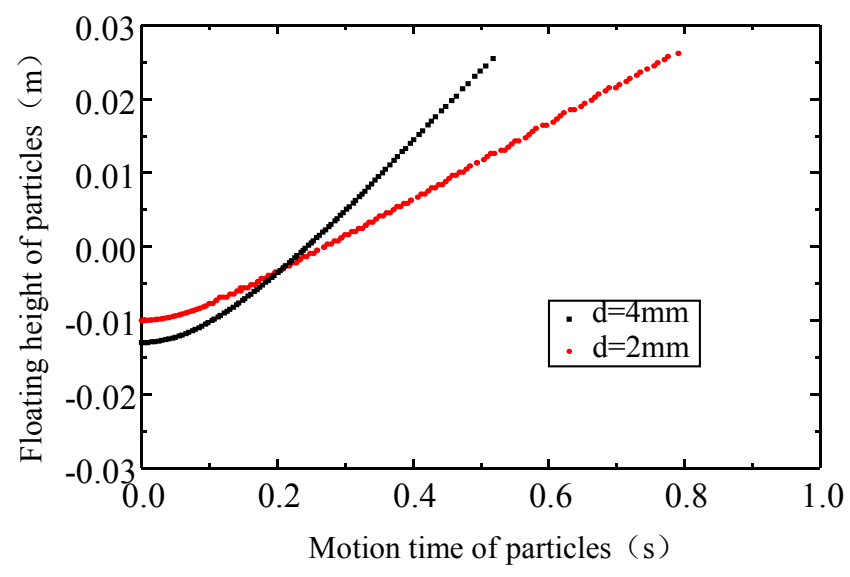

Fig.4 The variation of the floating height of particles with time

\section{Conclusions}

According to the trajectory of two particle cross principle, ignoring the acceleration process of particles, established two particle collision model, theoretical calculation of the model by using the Matlab software, and the simulation results of Fluent software simulation, the results show that the calculation results of the two methods in the horizontal direction of the particle movement and position almost the same, there are errors in the vertical direction, the overall good agreement.

\section{References}

[1] GuiNan. DEM-LES/DNS Coupling Simulation of Particle Collision in Complex Two-Phase Flows[D].Hangzhou: Zhejiang University, 2010.

[2] Yan-Peng, L.; Huan-Ran, W., Three-dimensional direct simulation of a droplet impacting into a solid sphere with low-impact energy. Can J Chem Eng. 2011,89(1): 83-91. 
[3] Kendoush A A, Sulaymon A H, Mohammed S A M, et.al. Experimental Evaluation of the Virtual Mass of Two Solid Spheres Accelerating in Fluids[J]. Exp.Therm.Fluid Sci,2007,31(7):813-823.

[4] Alizadeh, A.; McKenna, T. F. L.,Condensed mode cooling in ethylene polymerisation: Droplet evaporation. Macromolecular Symposia. 2013,333(1): 242-247.

[5] Wei Mingrui, Wen Hua, Liu Yongchang et.al. Modeling Study on Droplets Collision in Spray Process.Transactions of CSICE.2005, (6):518-523.

[6] Sun, J.; Zhou, Y.; Ren, C; Wang, J.; Yang, Y.' CFD simulation and experiments of dynamic parameters in gas-solid fluidized bed. Chem Eng ScL 2011,66(21): 4972-4982.

[7] Dehnavi, M. A.; Shahhosseini, S.; Hashemabadi, S. H.; Ghafelebashi, S. M.,CFD simulation of hydrodynamics and heat transfer in gas phase ethylene polymerization reactors. International Communications in Heat and Mass Transfer 2010,37(4): 437-442.

[8] Chen, X.-Z.; Luo, Z.-H.; Yan, W.-C.; Lu, Y.-H.; Ng, I. S.,Three-dimensional CFD-PBM coupled model of the temperature fields in fluidized-bed polymerization reactors. AIChE J. 2011, 57(12):3351-3366.

[9] Karimi, S.; Mansourpour, Z.; Mostoufi,N.; Sotudeh-Gharebagh, R" CFD-DEM study of temperature and concentration distribution in a polyethylene fluidized bed reactor. Particul Sci Technol.2011,29(2): 163-178.

[10] Rokkam, R. G.; Fox, R. O.; Muhle, M. E.s Computational fluid dynamics and electrostatic modeling of polymerization fluidized-bed reactors. Powder Technol. 2010,203(2): 109-124. 